\title{
Lei da Conservação das Massas: Experimentação e Contextualização
}

Maricélia Lucena Ferreira* (Graduanda em Química na Universidade Federal de Campina Grande-UFCG); Egle Katarinne Souza da Silva (Graduanda em Química na Universidade Federal de Campina Grande-UFCG); Luislândia Vieira de Figueiredo (Graduanda em Química na Universidade Federal de Campina Grande-UFCG); Luciano Leal de Morais Sales (Prof. Adjunto iv da Unidade Acadêmica de Ciências Exatas e da Natureza do Centro de Formação de Professores da Universidade Federal de Campina Grande-UFCG).

*E-mail: m/uc cena@hotmail.com

resumo:

A experimentação nas aulas do ensino de Química vem ocupando cada vez mais um espaço essencial no processo de ensino e aprendizagem. Como forma de investigação dessa metodologia de ensino, este trabalho apresenta resultados de uma pesquisa realizada em conjunto com a execução de uma atividade experimental, desenvolvidas com os alunos do $2^{\circ}$ ano do ensino médio da Escola Estadual de Ensino Fundamental e Médio Professor Manoel Mangueira Lima, localizada no município de Cajazeiras-PB. Objetivando contextualizar o estudo da Lei da Conservação das Massas, iniciaram-se as atividades com embasamento teórico reforçado através da leitura de periódicos relacionados ao assunto. Em seguida os discentes ministraram aulas práticas utilizando materiais alternativos de baixo custo enfocando o tema proposto em estudo. Antes e após as aulas ministradas, aplicou-se com os discentes da referida escola uma entrevista semiestruturada para consideração e a ponderação do conhecimento do alunado. Os resultados da pesquisa foram apresentados por meio de gráficos.

Visto que $100 \%$ da turma após as aulas ministradas e a demonstração da prática envolvendo a Lei da Conservação das Massas, conseguiu citar exemplos do cotidiano relacionados à mesma e que através da metodologia utilizada $66,70 \%$ dos discentes conseguiram explicar a frase "Na natureza nada se cria, nada se perde, tudo se transforma", com exemplos das aulas ministradas, podemos evidenciar que a aliança de aulas teóricas contextualizadas com aulas práticas só vem a contribuir com uma aprendizagem significativa por proporcionar a construção do conhecimento. Deste modo, o uso da experimentação em sala de aula acaba levando os discentes a se envolverem na construção do saber científico, tornando-os capazes de relacionar a Química com o cotidiano, mostrando-os que a Química está presente na vida diária de cada um. E para conseguir contextualizar o ensino de Química, é necessário levar em consideração o conhecimento prévio dos discentes, para assim introduzir de forma satisfatória o conhecimento teórico científico.

\section{palavras-chave:}

Lei da Conservação das Massas; Experimentação; Contextualização. 


\section{I NTRODUÇÃO}

Em razão do rápido desenvolvimento da ciência e da tecnologia que causaram um grande impacto na educação, tem-se firmado nas últimas décadas um consenso entre os educadores a respeito de melhorias na aprendizagem dos alunos nas escolas. O que depende de um eficaz esforço de pesquisa na área educacional, com um intuito de criar um conjunto de ideias sobre novas metodologias que possam vir a incentivar os alunos do ensino médio a se interessarem pelo conhecimento científico, em especial no que se refere à Química, compreendendo que este está relacionado ao seu cotidiano.

Diante dessa problemática, está convencionando-se entre professores e pesquisadores de química, que a utilização de aulas práticas auxilia na consolidação do conhecimento, além de ajudar no desenvolvimento cognitivo do aluno.

A experimentação significa uma forma de contextualizar o ensino e trazer a Química para mais próximo dos estudantes, permitindo uma articulação entre teoria e prática. Proporcionando um caráter motivador e lúdico, essencialmente vinculado aos sentidos com o objetivo de tornar o ensino de Química prazeroso, estimulante e satisfatório.

$\mathrm{Na}$ perspectiva de que a experimentação deve levar o aluno a pensar e refletir sobre os conhecimentos que estão sendo adquiridos, o presente trabalho tem por finalidade compreender qual a concepção do alunado a respeito do papel da experimentação nas aulas de química. Como também, procura averiguar de que maneira eles entendem o assunto da Lei da Conservação das Massas antes e após a execução de aulas discursiva e prática.

\section{METODOLOGI A}

A análise da coleta de dados foi realizada através de uma entrevista semiestruturada, aplicada com 46 alunos do $2^{\circ}$ ano do ensino médio da Escola Estadual de Ensino Fundamental e Médio Professor Manoel Mangueira Lima, situada no município de Cajazeiras. Inicialmente aplicou-se um questionário para se conhecer a realidade em que os alunos se encontravam em relação à Lei da Conservação das Massas. Em seguida iniciaram-se as aulas expositivas e discursivas utilizando-se de exemplos do cotidiano dos alunos relacionados ao tema, bem como uma abordagem a respeito da contextualização histórica e conceitos químicos ligados à temática. Dando continuidade realizou-se uma aula prática com materiais de baixo custo e fácil aquisição com o intuito de comprovar a referida lei.

Foram utilizados para a aula prática, os seguintes materiais:

- Uma balança de um prato

- Um pote plástico transparente

- Um pequeno copo de plástico descartável

- Bicarbonato de sódio $\left(\mathrm{NaHCO}_{3}\right)$

- Ácido acético (H3CCOOH = vinagre)

O objetivo do experimento é introduzir um reagente no pote plástico transparente e outro reagente no copo plástico, de forma que não haja contato entre os reagentes. Ao fechar o sistema, aferiu-se a massa. Em seguida, foi feito um movimento de forma que o pequeno copo plástico virasse, colocando os dois reagentes em contato. Observou-se a reação química e, em seguida, aferiu-se novamente a massa do sistema. A reação entre ácido acético e bicarbonato de sódio forneceu acetato de sódio em solução, gás carbônico e água, conforme a seguinte reação:

\section{CH3COOH + NaHCO3 ----- CH3COONa + CO2 + H20}

Após o experimento, os alunos debateram sobre o experimento e responderam a um segundo questionário da pesquisa, objetivando identificar como uma aula contextualizada e prática pode contribuir no desenvolvimento da aprendizagem do ensino de Química. 


\title{
3. RESULTADOS E DI SCUSSÃO
}

Com o intuito de compreender as características relevantes nas aulas e durante as atividades experimentais ou até após as mesmas, foi aplicada uma pesquisa semiestruturada com alunos do $2^{\circ}$ ano da escola supracitada. Assim, por meio da análise e interpretação das informações obtidas pertinentes às questões respondidas, os resultados foram representados graficamente através do editor de gráfico Excel.

Ao questionar se os alunos já tinham ouvido falar sobre a Lei da Conservação das Massas e quais exemplos referentes à mesma podem ser aplicados em seu cotidiano, obtemos as seguintes respostas, as quais foram divididas em duas partes. A primeira, antes da ministração das aulas teóricas e práticas, a segunda parte após as ministrações das aulas. De acordo com a primeira parte, 83,3\% dos alunos responderam que já tinham ouvido falar a respeito da Lei da Conservação das Massas, mas não lembravam suficientemente ao ponto de citarem exemplos de seu cotidiano. No entanto, $16,7 \%$ disseram nunca ter ouvido falar na referida lei, como mostra o Gráfico 01.

\section{OUVIRAM FALAR SOBRE A LEI DA CONSERVAÇÃO DAS MASSAS}

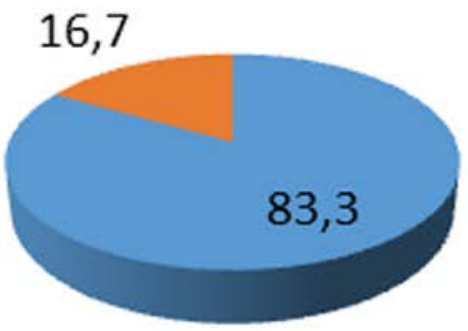

\author{
- JÁ OUVIRAM FALAR SOBRE LEI DA \\ CONSERVAÇÃO DAS MASSAS \\ NÃO OUVIRAM FALAR DA LEI DA \\ CONSERVAÇÃO DAS MASSA
}

Gráfico 01. Referente à pergunta se os alunos já tinham ouvido falar a respeito da Lei da Conservação das

Na segunda parte, todos foram unânimes em suas respostas, ou seja, após as ministrações das aulas teóricas e práticas, $100 \%$ dos alunos citaram exemplos de seu cotidiano relacionados à Lei da Conservação das Massas como mostra o Gráfico 02 e 03. Desse modo, de acordo com Bozzi (2009), o professor deve fazer o possível para proporcionar oportunidades aos alunos de maneira que eles possam realizar observações, colocar ideias em teste, coletar evidências e construir conclusões com base em evidências.
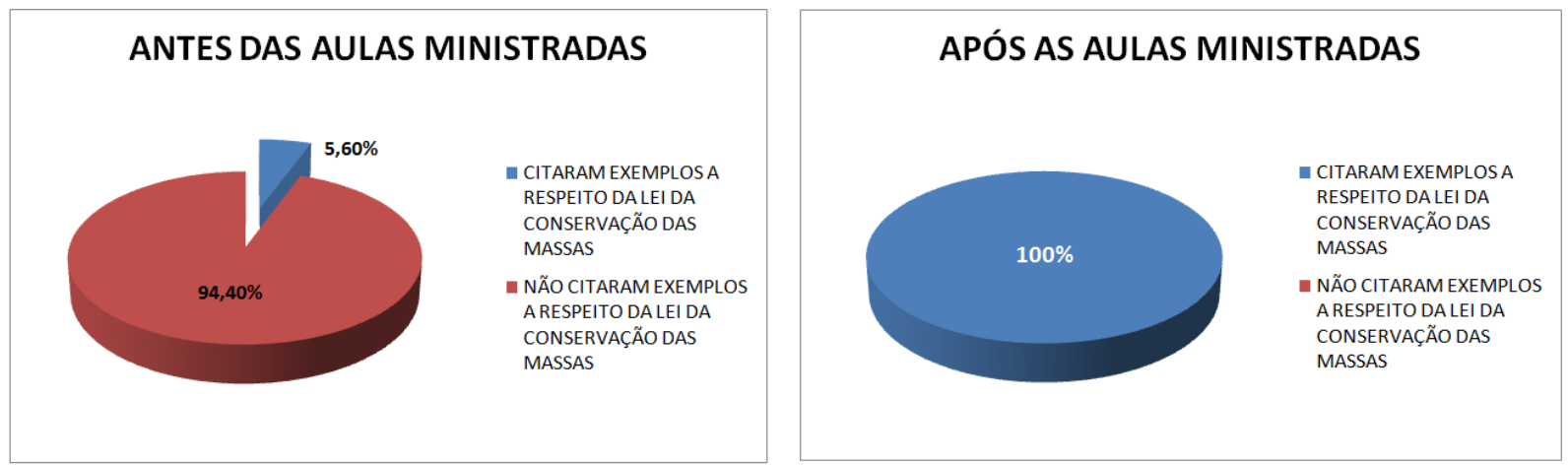

Gráficos 02 e 03 - Referente à citação de exemplos relacionados à Lei da Conservação das Massas, aplicado ao cotidiano dos alunos.

Os Gráficos 04 e 05 mostram as respostas dos alunos quando incitados a explicarem a frase "Na natureza nada se cria, nada se perde, tudo se transforma". Antes das aulas $89 \%$ da turma não responderam e os outros $11 \%$ deram respostas totalmente distante do verdadeiro sentido do enunciado. No entanto, após as aulas tanto teórica como as práticas, todos os alunos explicaram a frase citando exemplos relacionados com seu cotidiano, ou seja, $100 \%$ da turma. Isso demonstra como o ensino contextualizado é mais proveitoso, tendo em vista que o mesmo possibilita e facilita a absorção e compreensão dos conteúdos. 


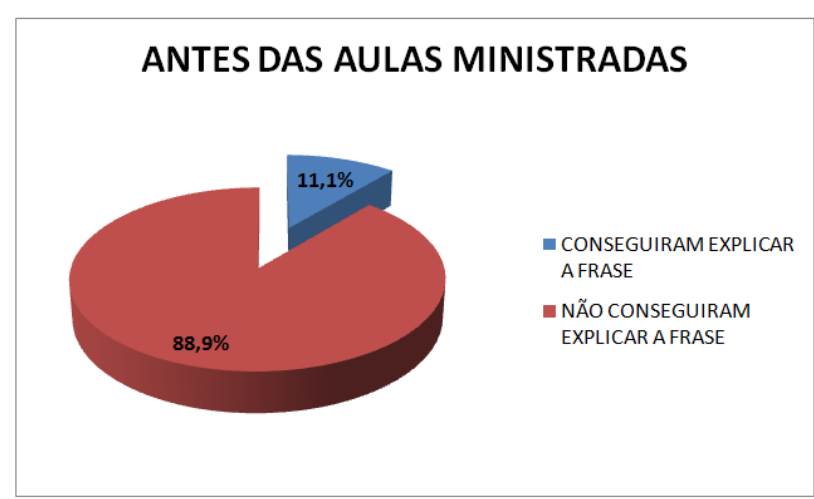

Gráfico 04. Referente à quando questionados a explicarem a frase "Na natureza nada se cria, nada se perde, tudo se transforma".

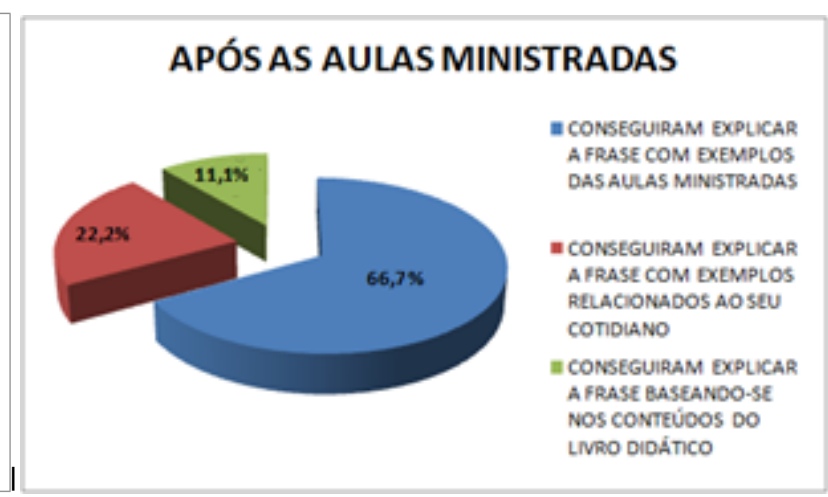

Gráfico 05. Referente às explicações dos estudantes a respeito da frase "Na natureza nada se cria, nada se perde, tudo se transforma".

Por último quando questionados sobre a importância da utilização de aulas experimentais demonstrado no Gráfico 6- Cerca de 45,3\% dos alunos afirmaram que a utilização da experimentação contribui para melhores explicações em relação aos conteúdos de Química. O que evidencia a importância de novas metodologias de ensino que instiguem os discentes a construir seu próprio conhecimento. Já outros $35,3 \%$, defenderam que este recurso proporciona associações entre a teoria e a prática, demonstrando que existe um elo entre as duas. Ainda 19,4\% dos discentes, responderam que facilita na compreensão dos conteúdos, onde se pode deduzir que por ser uma disciplina abstrata, a visualização de reações químicas através de demonstrações experimentais propicia a absorção dos mesmos.

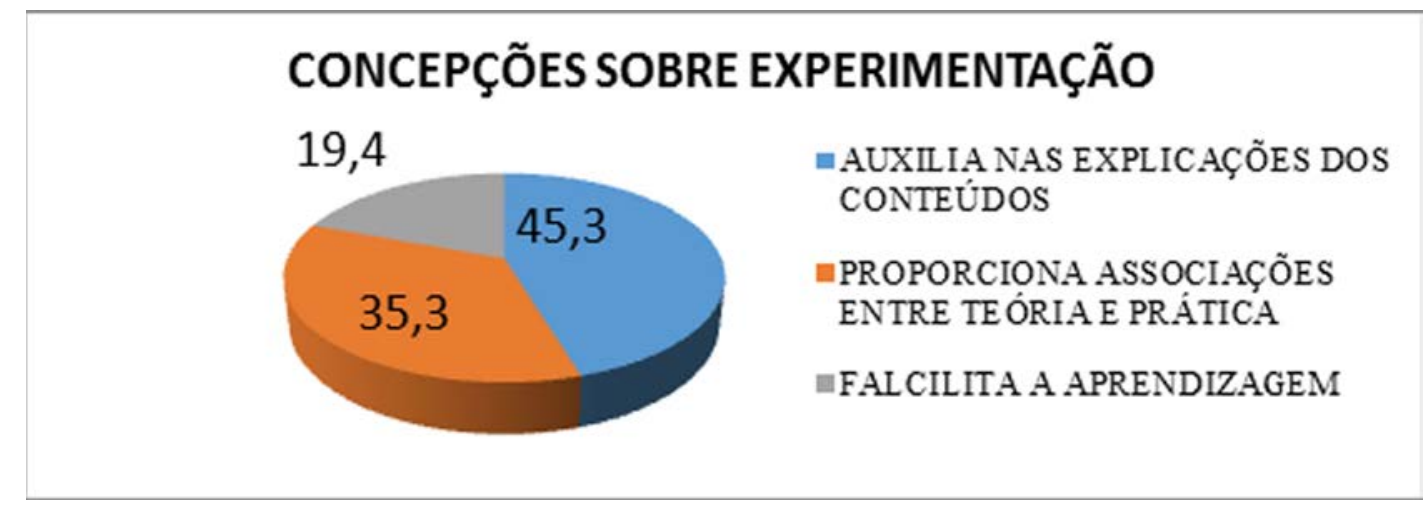

Gráfico 6- Mostra a Concepção dos Alunos a respeito de Aulas Experimentais.

\section{Conclusões}

Diante do exposto, verifica-se que a utilização de aulas contextualizadas seguidas de aulas práticas, além de favorecer a busca de explicações para fenômenos naturais, [...] constituirá uma excelente ocasião para que os alunos comecem a explicitar funcionalmente suas concepções espontâneas [...](CARVALHO; GILPÉREZ, 2006, P. 44). Esta metodologia também contribui para uma sistematização do conhecimento construído, e consequentemente, uma mudança conceitual. Como revelam os resultados aferidos na escola supracitada, que com a aplicação das atividades, participaram ativamente e declararam com mais propriedade aos questionários aplicados, o que comprova que o método reforçou o processo de ensino e aprendizagem dos discentes.

Conforme demonstra a pesquisa, $100 \%$ da turma conseguiu citar exemplos do cotidiano relacionados com a Lei da Conservação das Massas, visto que antes das aulas ministradas esse percentual era somente 5,6\% dos alunos. Guimarães (2009) afirma que em aulas expositivas o professor deve fazer uma análise das descobertas dos discentes, para assim abordar de forma significativa o conteúdo programático. Para tal, é preciso considerar o aprendizado da Química além do livro didático, construindo um saber com formato contextualizado. 
O professor pode considerar, em aulas expositivas, as descobertas dos aprendizes para trabalhar significativamente os conteúdos pretendidos, pois ao trabalhar com as dificuldades e explicações dos alunos ao fenômeno, ele aliará as concepções prévias aos novos conhecimentos. Não se trata de trabalhar a química que só existe no livro e para a escola. Ao utilizar a experimentação, associando os conteúdos curriculares ao que o educando vivenciou, o educador trabalhará de forma contextualizada, pois não é o problema proposto pelo livro ou a questão da lista de exercício, mas os problemas e as explicações construídas pelos atores do aprender diante de situações concretas. (Guimarães, 2009, p. 199).

Assim, as atividades experimentais para o ensino médio contribuem na compreensão do conhecimento químico por meio do contato do aluno com o objeto de estudo. Esse contato envolve o aluno auxiliando na assimilação dos conteúdos produzindo significado à ciência.

Law of Conservation of Mass : Experimentation and Contextualization

Abstract: Experimentation in Chemistry teaching is now occupying an increasingly essential space in the teaching and learning process. As a way of research regarding this teaching methodology, this paper presents results of a survey conducted in conjunction with the implementation of an experimental activity, developed with the second year students of the State High School Prof. Manoel Mangueira Lima, located in the municipality of Cajazeiras, PB, Brazil. In order to contextualize the study of the Law of Conservation of Mass, activities were started with theoretical foundation reinforced by reading journals related to the subject. Then the students ministered practical classes using alternative low-cost materials focusing on the theme under study. Before and after the ministering of the classes, a semi-structured interview was conducted with the students of this school in order to check and weight their knowledge. The results of this research were presented through graphs. Since 100 per cent of the students after the lectures and also after the practical demonstration involving the Law of Conservation of Mass were able to cite examples of everyday life related to the same subject, and, since through the methodology, 66.70 per cent of students explained the phrase "in nature nothing is created, nothing is lost, everything is transformed", with examples from the classes ministered, we could highlight that the joining of lectures with practical lessons only contribute to a significant learning, providing the construction of knowledge. In this way, the use of experimentation in the classroom ends up by taking the students to get involved in building scientific knowledge, making them able to relate the Chemistry with the everyday life, showing them that the Chemical is present in their daily life. And, to get the context of Chemistry Teaching, it is necessary to take into account the previous knowledge of the students, to introduce in a satisfactory way, the scientific and theoretical knowledge.

Keywords: Law of Conservation of Mass; Experimentation; Contextualization.

\section{Referências bibliográficas}

CARVAlHO, A. M. P. de; GI L-PEREZ, D. G. Formação de Professores de Ciências. 10 ed. Cortez. São Paulo: 2011.

DELIZOICOV, D.; ANGOTTI, J. A. Metodologia do Ensino de Ciências. 2. Ed. São Paulo: Cortez, 1994.

DELIZOICOV, D.; ANGOTTI, J. A.; PERNANBUCO, M. M. Ensino de Ciências: fundamentos e métodos. 3. Ed. São Paulo: Cortez, 2009. 
FILHO, J osé Ernane Carneiro Carvalho. Educação Científica na Perspectiva Bachelardiana: Ensino enquanto Formação. ENSAI O-Pesquisa em Educação em Ciências. Volume 28/ № 1, Julho de 2003.

GI L, Antonio Carlos. Métodos e técnicas de pesquisa social. São Paulo, Editora Atlas, 1987. Capítulo II. O questionário. Conceituação. Vantagens e limitações do questionário. A construção do questionário. p. 124132.

GIORDAN, Marcelo. O papel da Experimentação no ensino de Ciências. Publicado na revista Química Nova na Escola n. 10, nov. 1999.

GUIMARÃES, Cleidson Carneiro. Experimentação no Ensino de Química: Caminhos e Descaminhos Rumo à Aprendizagem Significativa, 2019. Disponível em: <http://webeduc. mec.gov.br/portaldoprofessor/quimica/sbq/QNEsc31_3/08-RSA-4107.pdf>. Acesso em: 15 nov. 2013.

MARTINS, Roberto de Andrade. MARTINS, Lilian Al-Chueyr Pereira. Lavoisier e a conservação da massa. Química nova na escola, 16(3) (1993).

SANTOS, Maria Eduarda Vaz Moniz. Mudança conceptual na sala de aula: um desafio pedagógico. Lisboa: Horizonte, 1991. 